\title{
Retinal Break Adjacent to the Optic Disc Causing Retinal Detachment in a Pathological Myopia
}

\author{
Noor Aniah $\mathrm{A}^{1,2}(\bowtie)$, Bastion $\mathrm{MLC}^{1}$, Mushawiahti $\mathrm{M}^{1}$ \\ ${ }^{1}$ Department of Ophthalmology, Faculty of Medicine, Universiti Kebangsaan Malaysia Medical Centre, \\ Jalan Yaacob Latif, Bandar Tun Razak, 56000 Cheras, Kuala Lumpur, Malaysia. \\ ${ }^{2}$ Ophthalmology Unit, Department of Surgery, Medical and Health Science Faculty, Universiti Putra \\ Malaysia, 43400 UPM Serdang, Selangor Darul Ehsan, Malaysia.
}

\begin{abstract}
Retinal detachment is one of the common complications of pathological myopia due to presence of retinal break. However, retinal break commonly occurs in the peripheral retina. This case report illustrates the rare incidence of retinal break adjacent to the optic disc, highlights the possible causes of poor visual outcome following surgical repair as well as the possible measures to treat the complications.
\end{abstract}

Keywords: Degenerative myopia, pathological myopia, retinal break, retinal detachment, subretinal fluid

\section{Correspondence:}

Noor Aniah Azmi. Department of Ophthalmology, Faculty of Medicine, Universiti Kebangsaan Malaysia Medical Centre, Jalan Yaacob Latif, Bandar Tun Razak, 56000 Cheras, Kuala Lumpur, Malaysia. Tel: +603-91457207 Fax: +603-91456733 E-mail: nooraniah@gmail.com

\section{Introduction}

Retinal detachment is one of the complications of pathological myopia. About $40 \%$ of retinal detachment occurs in myopic patients. Rhegmatogenous retinal detachment occurs as a result of a retinal break or lattice degeneration that is commonly associated with pathological myopia. Pathological myopia is defined as axial length more than $26 \mathrm{~mm}$ or refractive error of more than -6.0 diopters. The common sites of retinal break are in the peripheral retina, however, it can also occur around the optic disc. This case report illustrates the rare incidence of retinal break adjacent to the optic disc, highlights the possible causes of poor visual outcome following surgical repair as well as the possible measures to treat the complications.

\section{Case Report}

A 74-year-old Malay gentleman with underlying diabetes mellitus, hypertension and chronic myeloid leukemia presented with left eye blurring of vision, central scotoma and floaters for the past two months. $\mathrm{He}$ had no metamorphopsia and no recent ocular trauma. $\mathrm{He}$ is a known high myope and is bilaterally pseudophakic. His right axial length is $26.84 \mathrm{~mm}$ and left eye is $26.82 \mathrm{~mm}$. Visual acuity of the left eye during presentation was $6 / 36$ and N18. His intraocular pressure (IOP) and anterior segment was unremarkable. Posterior pole revealed retinal detachment surrounding the optic disc with vitreomacular traction (Fig. 1) with presence of submacular fluid detected on Optical Coherence Tomography (OCT) (Fig. 2). The patient refused surgery at the time of presentation and he came back after 2 months with worsening metamorphopsia, but visual acuity remained the same. Pars plana vitrectomy with internal limiting membrane peeling, endolaser and perfluoropropane $(\mathrm{C} 3 \mathrm{~F} 8)$ gas tamponade was performed. Intraoperatively, we noted that there was a small retinal break superior to the optic disc. Immediately after surgery, his visual acuity was counting finger (CF), which improved to $6 / 603$ months after. On OCT, there was persistent subretinal fluid involving the fovea and retinal detachment 
extending to the edges of the optic disc 360-degrees. Nine months after the surgery, his vision remained at $6 / 60$, however, the subretinal fluid was seen to be gradually reducing in these serial volume OCT scans.

\section{Discussion}

Retinal detachment (RD) is separation of the neurosensory layer of the retina from the retinal pigment epithelium. The separation is caused by a failure of the force that keeps the neurosensory retina (NSR) and retinal pigment epithelium (RPE) attached, and this results in accumulation of fluid beneath the retina, known as the subretinal fluid. About $40 \%$ of all RD cases occur in myopic eyes. In myopia, the axial length is increased and this can give rise to posterior vitreous detachment and retinal break (1). The most common site of retinal break is in the peripheral retina, particularly in the supero-temporal quadrant which accounts for $60 \%$ of cases because of the weak attachment of the vitreous. The inferonasal quadrant accounts for the fewest number of retinal break which is only $10 \%$ of cases. Retinal break adjacent to the optic disc is rare as the optic disc is of the sites for the strongest vitreous attachment. In 2004, Buller and Charles reported a similar case of peripapillary retinal break in a highly myopic patients, but it did not lead to

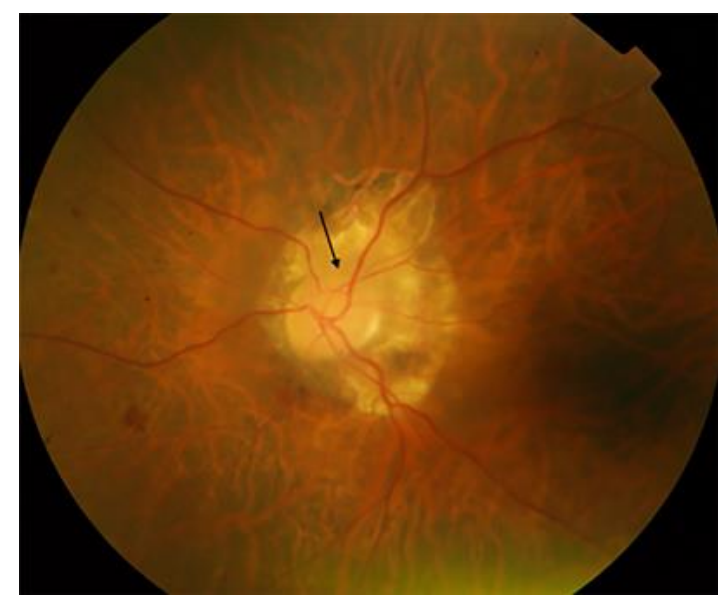

Figure 1: Fundus photo showing retinal detachment surrounding the optic disc.

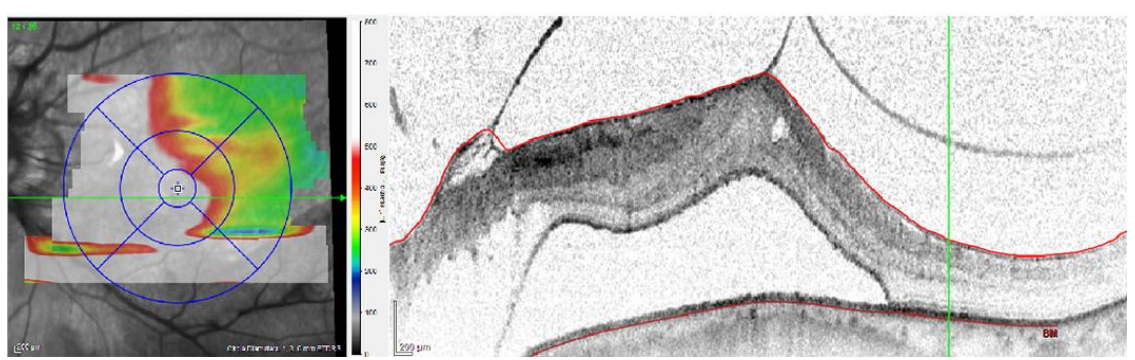

Figure 2: OCT image showing retinal detachment with subretinal fluid and vitreomacular traction (before surgery)

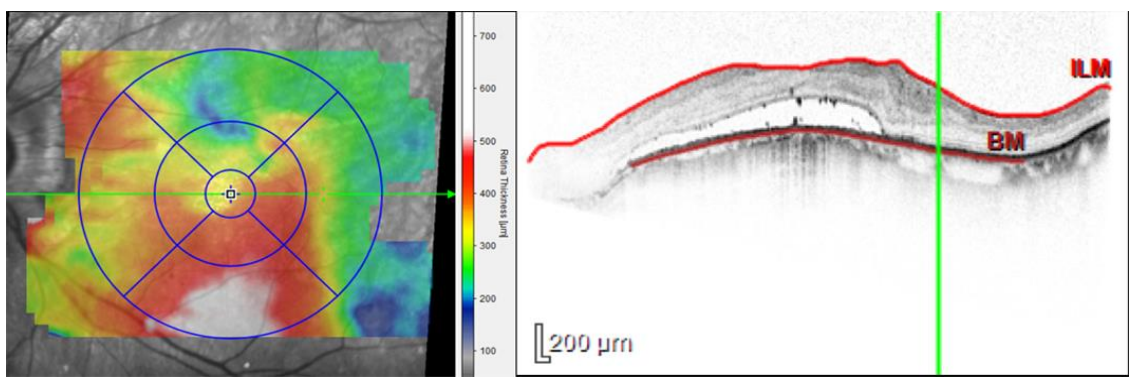

Figure 3: OCT image showing persistent subretinal fluid 3 months after surgery

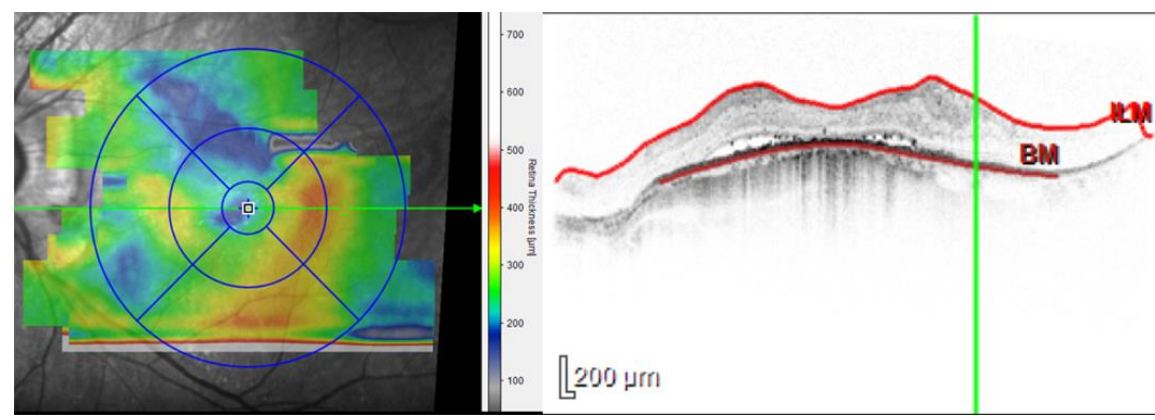

Figure 4: OCT image showing reduction of subretinal fluid 10 months after surgery 
retinal detachment due to absence of vitreoretinal traction in that particular case (2). In our case, the patient has high myopia with the axial length of more than $26 \mathrm{~mm}$ and it is one of the risk factor of developing retinal break due to thinning of the retina. The retinal break present adjacent to the optic disc becomes the entry point of the liquefied vitreous which causes the RRD. Vitreous synchisis is a common senile process.

Poor visual outcome following retinal detachment surgery can be due to several factors, which includes development of epiretinal membrane, persistent subretinal fluid following surgery, duration of retinal detachment before surgery and the anatomical changes of the retina following retinal detachment.

The duration of retinal detachment before surgery plays a role in determining the post-operative visual outcome. Based on an experimental retinal detachment in a cat model, a wave of photoreceptor apoptosis can occur as early as day 1 and peaks on day 3 (3). However, Ross and Kozy recommended that the surgical repair could be delayed up to one week with no adverse effects on the visual outcome in situations where macula was found to be detached for up to one week (4). In this patient, his surgery was delayed for more than 2 months and this could have contributed to his poor visual outcome after the surgery.

Persistent subretinal fluid in the fovea could be one of the causes of poor vision following surgical repair. The fovea contains densely-packed cones that are responsible for sharp central and near vision, hence, presence of fluid affects the alignment of these cones. The misalignment of the cones leads to metamorphopsia and poor vision. It was also postulated that subretinal fluid impairs the oxygen diffusion of the outer segment of the photoreceptors, hence, impaired metabolism that is vital for visual cycle process. As in this patient, the cause of persistent subretinal fluid could be due to chronicity of the condition leading to a more viscous fluid that are more difficult to be reabsorbed. However, Seo et al. investigated the final best-corrected vision 12 months post-surgery in patients who underwent scleral-buckle for RRD with macula-off (5). He found that the presence of persistent SRF does not determine the final visual outcome after 1 year following surgery as his study showed no significant difference of BCVA after 1 year in the group with no persistent SRF and the group with persistent SRF. As for this patient, his BCVA improved after 3 months despite the presence of SRF and it remained the same after 1 year of surgery.

\section{Conclusion}

This reports illustrates that retinal break can occur around the optic disc, which is the location of the strongest vitreous attachment. The duration of retinal detachment before surgery plays a role in determining the final visual outcome. The surgical repair can be delayed up to one week as during detachment. The presence of persistent SRF may lead to slow recovery of the final visual outcome.

\section{References}

1. Kanski Jack J. Clinical Ophthalmology: A Systematic Approach, 6th ed. Edinburgh: Butterworth-Heinemann/Elsevier, 2007.

2. Buller AJ, Charles SJ. Retinal tear arising adjacent to the optic disc: a case report. Eye (Lond) 2004; 18(9): 945-6.

3. Cook B, Lewis GP, Fisher SK, Adler R. Apoptotic photoreceptor degeneration in experimental retinal detachment. Invest Ophthalmol Vis Sci 1995; 36(6): 990-6.

4. Ross WH, Kozy DW. Visual recovery in maculaoff rhegmatogenous retinal detachments. Ophthalmology. 1998; 105(11): 2149-53.

5. Seo JH, Woo SJ, Park KH, Yu YS, Chung H. Influence of persistent submacular fluid on visual outcome after successful scleral buckle surgery for macula-off retinal detachment. Am J Ophthalmol 2008; 145(5): 915-22. 